\title{
SYMBOLIC COMPUTATIONAL MODELS FOR INTUITIONISTIC LINGUISTIC INFORMATION
}

\author{
PHAM HONG PHONG ${ }^{1}$, BUI CONG CUONG ${ }^{2}$ \\ ${ }^{1}$ Faculty of Information Technology, National University of Civil Engineering; \\ phphong84@yahoo.com \\ ${ }^{2}$ Institute of Mathematics, Vietnam Academy of Science and Technology; bccuong@gmail.com
}

\begin{abstract}
In 2014, the notion of intuitionistic linguistic labels was first introduced. In this paper, we develop two symbolic computational models for intuitionistic linguistic labels. Various operators are proposed, and their properties are also examined. Then, an application to group decision making using intuitionistic linguistic preference relations is discussed.

Keywords. linguistic aggregation operator, linguistic symbolic computational model, intuitionistic linguistic label, group decision making, linguistic preference relation
\end{abstract}

\section{INTRODUCTION}

\subsection{Group decision making problem under linguistic information}

Decision making is the process of choosing alternative(s) among several alternatives based on the assessments given by decision makers (DMs). The uncertainty and the fuzziness of human thought result in decision making with linguistic information in a wide variety of practical problems. In 2000, Herrera and Herrera-Viedma [14] proposed the solution scheme for solving group decision making (GDM) problems under linguistic information:

(1) Specification of the linguistic term set with its semantic. In this step, the linguistic variable [30] or linguistic expression domain with a semantic is established to provide the evaluations about alternatives according to the different criteria.

(2) Choice of the appropriate aggregation operators of linguistic information. This step depends on the characteristics of the problem and how we represent linguistic terms of the linguistic variable.

(3) Choice of the best alternative(s). It consists of two phases:

(a) Aggregation phase: It consists of obtaining overall linguistic assessments on the alternatives by aggregating the assessments provided according to all the criteria by means of the chosen aggregation operator of linguistic information.

(b) Exploitation phase: It consists of establishing a rank ordering the alternatives according to the overall assessments for choosing the best alternative(s).

Many aggregations which aggregate linguistic information were developed. They are classified into four groups $[15,16]$ :

(c) 2015 Vietnam Academy of Science \& Technology 
(1) Linguistic computational model based on membership functions (type-1 fuzzy sets). Linguistic terms are seen as fuzzy numbers. Using Extension Principle, the result of the aggregation is also a fuzzy number. Then, an approximation function is utilized to associate this result with a particular label.

(2) Linguistic computational model based on type-2 fuzzy sets. This model is an improvement of the previous one by replacing the type- 1 representation by type- 2 representation. According to this approach, computational processes make use the interval type-2 fuzzy sets (a particular kind of the type-2 fuzzy sets) which maintain the uncertainty but reduce the computational performance.

(3) Linguistic symbolic computational models based on ordinal scales. This model has been widely used in computing with words due to its simplicity and high interpretability. It has two characteristics:

- A finite and totally ordered discrete label set is used to represent the information;

- The operators, according to this model, perform calculations on the indices of the linguistic labels.

(4) Linguistic symbolic computational models based on 2-tuple representation. Linguistic information is presented as a pair of values, called linguistic 2-tuple, $(s, \alpha)$, where $s$ is a linguistic label and $\alpha$ is a numeric value representing a Symbolic Translation. This model makes calculations with linguistic labels easily and without loss of information.

Tanino $[19,20]$ represented the information about the set of alternatives in three different ways: as a preference ordering of the alternatives, as a fuzzy preference relation, and as a utility function. Hence, obtaining a uniform representation of the evaluations must be the first step of the resolution process of the GDM problem. Chiclana, Herrera and Herrera-Viedma [4] showed that a preference ordering of the alternatives and a utility function can be converted to achieve a preference relation. So, preference relations have been frequently considered in the GDM problem: Delgado et al. [8] studied the situations in which DMs provide their preference information by using linguistic labels, $\mathrm{Xu}[26]$ introduced the concept of intuitionistic preference relations and their application in GDM, Zhang [31] proposed GDM with 2-tuple intuitionistic fuzzy linguistic preference relations, Xia and $\mathrm{Xu}[21]$ developed an approach to GDM problem based on the hesitant fuzzy preference relations, ...

\subsection{Intuitionistic linguistic label set}

Motivated by Atanassov's intuitionistic fuzzy set theory [1,2], we defined the intuitionistic linguistic label $[5,17]$. The novel definition is useful in situations when experts' opinions are given as pairs of linguistic labels. For each pair of linguistic labels, the first label expresses the membership and the second expresses the nonmembership (of an element in a set).

For example, the relation between a car $x$ and the set of good cars $A$ can be expressed by two components: (linguistic) membership component $\mu_{A}(x)=$ very_likely, and (linguistic) nonmembership component $\nu_{A}(x)=$ impossibly. So the pair (very_likely, impossibly) is an intuitionistic linguistic label expressing this relation. 
Some order relations for intuitionistic linguistic information were developed: membership-based order relation and nonmembership-based order relation [17], score and confidence-based order relation [5]. Using order relations, intuitionistic linguistic max and min [17], intuitionistic linguistic weighted median [17], intuitionistic linguistic fuzzy relations and max-min composition of these relations [5] were introduced.

\subsection{The organization of the paper}

The rest of the paper is organized as follows. Section 2 gives an overview of linguistic symbolic computational models as well as intuitionistic label set. Sections 3 and 4 are devoted to present the contributions of the paper: two linguistic symbolic computational models for intuitionistic linguistic information. Section 5 proposes a refined algorithm, which use the new operators, for GDM problem under intuitionistic fuzzy linguistic preference relations. Section 6 draws a conclusion.

\section{PRELIMINARIES}

\subsection{Linguistic symbolic computational models}

\subsubsection{Linguistic symbolic computational model for discrete label set}

Yager [28] presented the linguistic information using a finite and totally ordered discrete label set:

$$
S=\left\{s_{0}, s_{1}, \ldots, s_{g}\right\},
$$

such that $s_{i} \geq s_{j}$ iff $i \geq j$. In order to aggregate linguistic labels, the classical operators (maximum, minimum and negation) are used:

$$
\max \left(s_{i}, s_{j}\right)=s_{i} \text { if } i \geq j \quad ; \quad \min \left(s_{i}, s_{j}\right)=s_{i} \text { if } i \leq j \quad ; \quad \operatorname{neg}\left(s_{i}\right)=s_{g-i} .
$$

Example 2.1. A set of seven linguistic labels could be [3]:

$$
\begin{aligned}
S= & \left\{s_{0}=\text { none, } s_{1}=\text { very_low }, s_{2}=\text { low }, s_{3}=\text { medium },\right. \\
& \left.s_{4}=\text { high }, s_{5}=\text { very_high, } s_{6}=\text { perfect }\right\} .
\end{aligned}
$$

Using these classical operators, many operators were developed: ordinal ordered weighted averaging (OOWA) operator [29], linguistic weighted disjunction (LWD) and conjunction (LWC) operators [10], ordinal hybrid aggregation (OHA) operator [22], ... .

The convex combination of linguistic labels [7] was also employed in this computational model. It provides a wider range of aggregation operators. An aggregation operator, which uses the convex combination of linguistic labels, can be interpreted as $[11,12]$ :

$$
S^{n} \stackrel{\mathrm{C}}{\rightarrow}[0, g] \stackrel{\operatorname{app}(\cdot)}{\longrightarrow}\{0, \ldots, g\} \rightarrow S,
$$

where $\mathrm{C}$ is an operator which directly acts over the label indexes, and app $(\cdot)$ is an approximation function used to obtain an index associated with a linguistic label. Some well known operators of this type are linguistic ordered weighted averaging $\left(\mathrm{LOWA}_{1}\right)[9]$, induced linguistic ordered weighted averaging $\left(\mathrm{ILOWA}_{1}\right)[10]$, linguistic weighted averaging $\left(\mathrm{LWA}_{1}\right)$ [13], linguistic weighted OWA (LWOWA) operators [18], ... .

The finite and totally ordered discrete label set, $S$, is also described as follows ( $g$ is an even positive integer): 
- Subscript-symmetric linguistic evaluation scale [6]:

$$
S=\left\{s_{\alpha} \mid \alpha=-\frac{g}{2}, \ldots,-1,0,1, \ldots, \frac{g}{2}\right\} .
$$

- Multiplicative linguistic evaluation scale [23]:

$$
S=\left\{s_{\alpha} \mid \alpha=\frac{1}{\frac{g}{2}+1}, \ldots, \frac{1}{2}, 1,2, \ldots, \frac{g}{2}+1\right\}
$$

-... .

Similar to the linguistic evaluation scale in (1), aggregation operators were also developed for scales in (2-3).

\subsubsection{Linguistic Symbolic Computational Model for Continuous Label Set}

$\mathrm{Xu}[27]$ introduced the computational model to improve the accuracy in processes of linguistic aggregation by extending the subscript-symmetric linguistic evaluation scale (2) to the continuous linguistic one, $\bar{S}=\left\{s_{\alpha} \mid \alpha \in[-t, t]\right\}$, where $t(t>g)$ is a sufficiently large positive integer. If $s_{\alpha} \in S$, then $s_{\alpha}$ is called an original linguistic label; otherwise, an extended (or virtual) linguistic label. The classical operators maximum, minimum and negation were defined similarly to the those of $S$.

Example 2.2. [24] The discrete label set $S=\left\{s_{-3}, s_{-2}, s_{-1}, s_{0}, s_{1}, s_{2}, s_{3}\right\}$ (the set of original linguistic terms) is extended to a continuous one $\bar{S}=\left\{s_{\alpha} \mid \alpha \in[-3,3]\right\}$. The label $s_{-0.3} \in \bar{S}$, for example, is a virtual linguistic label.

The operators which aggregate continuous labels were developed [25]: linguistic averaging (LA), linguistic weighted averaging $\left(\mathrm{LWA}_{2}\right)$, linguistic ordered weighted averaging $\left(\mathrm{LOWA}_{2}\right)$, induced linguistic OWA (ILOWA 2 ) operators, ... .

\subsection{Intuitionistic label set}

Definition 2.1. [5, 17] An intuitionistic linguistic label is defined as a pair of linguistic labels $\left(s_{i}, s_{j}\right) \in S^{2}$ such that $i+j \leq g$, where $S=\left\{s_{0}, s_{1}, \ldots, s_{g}\right\}$ is the linguistic label set; $s_{i}$ and $s_{j} \in S$ respectively define the degree of membership and the degree of nonmembership of an object in a set. The set of all intuitionistic linguistic labels is denoted as $\tilde{S}$. Two intuitionistic linguistic labels $\tilde{a}=\left(s_{i}, s_{j}\right), \tilde{b}=\left(s_{p}, s_{q}\right)$ are termed to be equal, denoted by $\tilde{a}=\tilde{b}$, if $s_{i}=s_{p}$ and $s_{j}=s_{q}$.

Definition 2.2. [5] For each $\tilde{a}=\left(s_{i}, s_{j}\right) \in \tilde{S}$, the score, $\operatorname{SC}(\tilde{a})$, and the confidence, $\operatorname{CF}(\tilde{a})$, of $\tilde{a}$ are respectively defined as follows:

$$
\mathrm{SC}(\tilde{a})=i-j \quad ; \quad \mathrm{CF}(\tilde{a})=i+j .
$$

Definition 2.3. [5] For all $\tilde{a}, \tilde{b} \in \tilde{S}$, we define:

$$
\tilde{a} \geq \tilde{b} \Leftrightarrow\left[\begin{array}{l}
\operatorname{SC}(\tilde{a})>\operatorname{SC}(\tilde{b}) \\
\left\{\begin{array}{l}
\operatorname{SC}(\tilde{a})=\operatorname{SC}(\tilde{b}) \\
\operatorname{CF}(\tilde{a}) \geq \operatorname{CF}(\tilde{b})
\end{array} ; \tilde{a}>\tilde{b} \Leftrightarrow\left\{\begin{array}{l}
\tilde{a} \geq \tilde{b} \\
\tilde{a} \neq \tilde{b}
\end{array} .\right.\right.
\end{array}\right.
$$


Table 1: The $\tilde{S}$ is reordered such that $\tilde{s}_{0} \leq \tilde{s}_{1} \ldots \leq \tilde{s}_{G}(G=27)$

\begin{tabular}{|c|c|c|c|c|c|c|c|}
\hline & $\mathrm{CF}=0$ & $\mathrm{CF}=1$ & $\mathrm{CF}=2$ & $\overline{C F}=3$ & $\mathrm{CF}=4$ & $\mathrm{CF}=5$ & $\overline{C F}=6$ \\
\hline $\mathrm{SC}=-6$ & & & & & & & $\tilde{s}_{0}=\left(s_{0}, s_{6}\right)$ \\
\hline $\mathrm{SC}=-5$ & & & & & & $\tilde{s}_{1}=\left(s_{0}, s_{5}\right)$ & \\
\hline $\mathrm{SC}=-4$ & & & & & $\tilde{s}_{2}=\left(s_{0}, s_{4}\right)$ & & $\tilde{s}_{3}=\left(s_{1}, s_{5}\right)$ \\
\hline $\mathrm{SC}=-3$ & & & & $\tilde{s}_{4}=\left(s_{0}, s_{3}\right)$ & & $\tilde{s}_{5}=\left(s_{1}, s_{4}\right)$ & \\
\hline $\mathrm{SC}=-2$ & & & $\tilde{s}_{6}=\left(s_{0}, s_{2}\right)$ & & $\tilde{s}_{7}=\left(s_{1}, s_{3}\right)$ & & $\tilde{s}_{8}=\left(s_{2}, s_{4}\right)$ \\
\hline $\mathrm{SC}=-1$ & & $\tilde{s}_{9}=\left(s_{0}, s_{1}\right)$ & & $\tilde{s}_{10}=\left(s_{1}, s_{2}\right)$ & & $\tilde{s}_{11}=\left(s_{2}, s_{3}\right)$ & \\
\hline $\mathrm{SC}=0$ & $\tilde{s}_{12}=\left(s_{0}, s_{0}\right)$ & & $\tilde{s}_{13}=\left(s_{1}, s_{1}\right)$ & & $\tilde{s}_{14}=\left(s_{2}, s_{2}\right)$ & & $\tilde{s}_{15}=\left(s_{3}, s_{3}\right)$ \\
\hline $\mathrm{SC}=1$ & & $\tilde{s}_{16}=\left(s_{1}, s_{0}\right)$ & & $\tilde{s}_{17}=\left(s_{2}, s_{1}\right)$ & & $\tilde{s}_{18}=\left(s_{3}, s_{2}\right)$ & \\
\hline $\mathrm{SC}=2$ & & & $\tilde{s}_{19}=\left(s_{2}, s_{0}\right)$ & & $\tilde{s}_{20}=\left(s_{3}, s_{1}\right)$ & & $\tilde{s}_{21}=\left(s_{4}, s_{2}\right)$ \\
\hline $\mathrm{SC}=3$ & & & & $\tilde{s}_{22}=\left(s_{3}, s_{0}\right)$ & & $\tilde{s}_{23}=\left(s_{4}, s_{1}\right)$ & \\
\hline $\mathrm{SC}=4$ & & & & & $\tilde{s}_{24}=\left(s_{4}, s_{0}\right)$ & & $\tilde{s}_{25}=\left(s_{5}, s_{1}\right)$ \\
\hline $\mathrm{SC}=5$ & & & & & & $\tilde{s}_{26}=\left(s_{5}, s_{0}\right)$ & \\
\hline $\mathrm{SC}=6$ & & & & & & & $\tilde{s}_{27}=\left(s_{6}, s_{0}\right)$ \\
\hline
\end{tabular}

Example 2.3. Consider the linguistic label set of seven labels $S=\left\{s_{0}, s_{1}, s_{2}, s_{3}, s_{4}, s_{5}, s_{6}\right\}$. The corresponding intuitionistic label set is $\tilde{S}=\left\{\left(s_{i}, s_{j}\right) \in S^{2} \mid i+j \leq 6\right\}$. Using the relation " $\geq$ " (Definition 2.3), $\tilde{S}$ can be ordered as in Table 1 .

Definition 2.4. [5] Let $\left\{\tilde{a}_{1}, \ldots, \tilde{a}_{n}\right\}$ be a collection of intuitionistic linguistic labels in $\tilde{S}$, the max, min operators are defined as follows:

$$
\max \left(\tilde{a}_{1}, \ldots, \tilde{a}_{n}\right)=\tilde{b}_{1} \quad ; \quad \min \left(\tilde{a}_{1}, \ldots, \tilde{a}_{n}\right)=\tilde{b}_{n},
$$

where $\tilde{b}_{j}$ is the $j$-th largest of the $\tilde{a}_{i}$.

\section{AGGREGATION OPERATORS FOR DISCRETE INTUITIONISTIC LABEL SET}

\subsection{More on intuitionistic linguistic max-min operators}

Let $\left\{\tilde{a}_{1}, \ldots, \tilde{a}_{n}\right\}$ be a collection of intuitionistic linguistic arguments in $\tilde{S}$. In order to find $\max \left(\tilde{a}_{1}, \ldots, \tilde{a}_{n}\right)$ and $\min \left(\tilde{a}_{1}, \ldots, \tilde{a}_{n}\right)$, some notations are used:

$$
\begin{aligned}
& \arg \max _{\mathrm{SC}}\left(\tilde{a}_{1}, \ldots, \tilde{a}_{n}\right)=\left\{\tilde{a} \mid \mathrm{SC}(\tilde{a})=\max _{k=1, \ldots, n}\left\{\operatorname{SC}\left(\tilde{a}_{k}\right)\right\}\right\} ; \\
& \arg \min _{\mathrm{SC}}\left(\tilde{a}_{1}, \ldots, \tilde{a}_{n}\right)=\left\{\tilde{a} \mid \mathrm{SC}(\tilde{a})=\min _{k=1, \ldots, n}\left\{\mathrm{SC}\left(\tilde{a}_{k}\right)\right\}\right\} ; \\
& \arg \max _{\mathrm{CF}}\left(\tilde{a}_{1}, \ldots, \tilde{a}_{n}\right)=\left\{\tilde{a} \mid \mathrm{CF}(\tilde{a})=\max _{k=1, \ldots, n}\left\{\mathrm{CF}\left(\tilde{a}_{k}\right)\right\}\right\} ; \\
& \arg \min _{\mathrm{CF}}\left(\tilde{a}_{1}, \ldots, \tilde{a}_{n}\right)=\left\{\tilde{a} \mid \mathrm{CF}(\tilde{a})=\min _{k=1, \ldots, n}\left\{\mathrm{CF}\left(\tilde{a}_{k}\right)\right\}\right\} .
\end{aligned}
$$

Theorem 3.1. For all collections of intuitionistic linguistic labels $\left\{\tilde{a}_{1}, \ldots, \tilde{a}_{n}\right\}$ in $\tilde{S}$, we have:

(A1) $\arg \max _{\mathrm{CF}}\left(\arg \max _{\mathrm{SC}}\left(\tilde{a}_{1}, \ldots, \tilde{a}_{n}\right)\right)$ contains a unique element $\tilde{a}^{*}$, and

$$
\max \left(\tilde{a}_{1}, \ldots, \tilde{a}_{n}\right)=\tilde{a}^{*}
$$

(A2) $\arg \min _{\mathrm{CF}}\left(\arg \min _{\mathrm{SC}}\left(\tilde{a}_{1}, \ldots, \tilde{a}_{n}\right)\right)$ contains a unique element $\tilde{a}_{*}$, and

$$
\min \left(\tilde{a}_{1}, \ldots, \tilde{a}_{n}\right)=\tilde{a}_{*} .
$$


Proof. Let us consider $\tilde{a}^{*} \in \arg \max _{\mathrm{CF}}\left(\arg \max _{\mathrm{SC}}\left(\tilde{a}_{1}, \tilde{a}_{2}, \ldots, \tilde{a}_{n}\right)\right)$. With the assumption that $\arg \max _{\mathrm{SC}}\left(\tilde{a}_{1}, \ldots, \tilde{a}_{n}\right)=\left\{\tilde{b}_{1}, \ldots, \tilde{b}_{m}\right\}$, we get $\tilde{a}^{*} \in \arg \max _{\mathrm{CF}}\left(\tilde{b}_{1}, \ldots, \tilde{b}_{m}\right)$. Thus, $\operatorname{SC}\left(\tilde{a}^{*}\right)=$ $\max \left\{\operatorname{SC}\left(\tilde{a}_{1}\right), \ldots, \operatorname{SC}\left(\tilde{a}_{n}\right)\right\}$, and $\operatorname{CF}\left(\tilde{a}^{*}\right)=\max \left\{\mathrm{CF}\left(\tilde{b}_{1}\right), \ldots, \mathrm{CF}\left(\tilde{b}_{m}\right)\right\}$. Because each pair of score and confidence determines a unique intuitionistic linguistic label, $\tilde{a}^{*}$ is the unique element of $\arg \max _{\mathrm{CF}}\left(\arg \max _{\mathrm{SC}}\left(\tilde{a}_{1}, \ldots, \tilde{a}_{n}\right)\right)$.

For each $\tilde{a} \in\left\{\tilde{a}_{1}, \ldots, \tilde{a}_{n}\right\}$, there are two cases:

- If $\tilde{a} \notin\left\{\tilde{b}_{1}, \ldots, \tilde{b}_{m}\right\}$, then $\operatorname{SC}(\tilde{a})<\operatorname{SC}\left(\tilde{a}^{*}\right)$.

- If $\tilde{a} \in\left\{\tilde{b}_{1}, \ldots, \tilde{b}_{m}\right\}$, then $\operatorname{SC}(\tilde{a})=\operatorname{SC}\left(\tilde{a}^{*}\right)$ and $\mathrm{CF}(\tilde{a}) \leq \mathrm{CF}\left(\tilde{a}^{*}\right)$.

By definition 2.3, $\tilde{a} \leq \tilde{a}^{*}$. So, $\max \left(\tilde{a}_{1}, \ldots, \tilde{a}_{n}\right)=\tilde{a}^{*}$. The remainder is similar.

Example 3.1. Consider $\tilde{a}_{1}=\left(s_{0}, s_{4}\right), \tilde{a}_{2}=\left(s_{2}, s_{4}\right), \tilde{a}_{3}=\left(s_{3}, s_{1}\right), \tilde{a}_{4}=\left(s_{1}, s_{5}\right), \tilde{a}_{5}=\left(s_{4}, s_{2}\right)$. We have:

$$
\begin{aligned}
\max \left(\tilde{a}_{1}, \ldots, \tilde{a}_{n}\right) & =\arg \max _{\mathrm{CF}}\left(\arg \max _{\mathrm{SC}}\left(\tilde{a}_{1}, \tilde{a}_{2}, \tilde{a}_{3}, \tilde{a}_{4}, \tilde{a}_{5}\right)\right) \\
& =\arg \max _{\mathrm{CF}}\left(\tilde{a}_{3}, \tilde{a}_{5}\right)=\tilde{a}_{5} \\
\min \left(\tilde{a}_{1}, \ldots, \tilde{a}_{n}\right) & =\arg \min _{\mathrm{CF}}\left(\arg \min _{\mathrm{SC}}\left(\tilde{a}_{1}, \tilde{a}_{2}, \tilde{a}_{3}, \tilde{a}_{4}, \tilde{a}_{5}\right)\right) \\
& =\arg \min _{\mathrm{CF}}\left(\tilde{a}_{1}, \tilde{a}_{4}\right)=\tilde{a}_{4} .
\end{aligned}
$$

\subsection{Intuitionistic linguistic aggregation median operators}

In this sub-section, we define some operators on intuitionistic linguistic labels by using the relation " $\geq$ " (Definition 2.3). From now on, $W=\left(w_{1}, \ldots, w_{n}\right)$ denotes a weight vector such that $w_{i} \geq 0$, and $\sum_{i=1}^{n} w_{i}=1$.

Definition 3.1. Let $\left\{\tilde{a}_{1}, \ldots, \tilde{a}_{n}\right\}$ be a collection of intuitionistic linguistic labels in $\tilde{S}$, and $\left\{\tilde{b}_{1}, \ldots, \tilde{b}_{n}\right\}$ be a permutation of $\left\{\tilde{a}_{1}, \ldots, \tilde{a}_{n}\right\}$ such that $\tilde{b}_{1} \geq \tilde{b}_{2} \cdots \geq \tilde{b}_{n}$. We define:

(1) Intuitionistic linguistic median operator:

$$
\operatorname{iMed}\left(\tilde{a}_{1}, \ldots, \tilde{a}_{n}\right)=\left\{\begin{array}{ccc}
\tilde{b}_{\frac{n+1}{2}} & \text { if } & n \text { is odd } \\
\tilde{b}_{\frac{n}{2}} & \text { if } & n \text { is even }
\end{array}\right.
$$

(2) Intuitionistic linguistic weighted median: consider the collection $\left\{\left(w_{1}, \tilde{a}_{1}\right), \ldots,\left(w_{n}, \tilde{a}_{n}\right)\right\}$, where $W=\left(w_{1}, \ldots, w_{n}\right)$ is a weight vector, $w_{i}$ is associated weight of $\tilde{a}_{i}$. We assume that $\left\{\left(u_{1}, \tilde{b}_{1}\right), \ldots,\left(u_{n}, \tilde{b}_{n}\right)\right\}$ is the ordered collection of $\left\{\left(w_{1}, \tilde{a}_{1}\right), \ldots,\left(w_{n}, \tilde{a}_{n}\right)\right\}$ such that $u_{j}$ is the weight that is associated with the $a_{i}$ that becomes $b_{j}$. Let $T_{i}=\sum_{j=1}^{i} u_{j}$, intuitionistic linguistic weighted median (iLWM) operator is defined as:

$$
\operatorname{iLWM}\left(\left(w_{1}, \tilde{a}_{1}\right), \ldots,\left(w_{n}, \tilde{a}_{n}\right)\right)=\tilde{b}_{k},
$$

where $k$ is the smallest integer such that $T_{k}$ is greater than or equal to 0.5 . 
Example 3.2. Consider $\tilde{a}_{1}=\left(s_{0}, s_{4}\right), \tilde{a}_{2}=\left(s_{2}, s_{4}\right), \tilde{a}_{3}=\left(s_{3}, s_{1}\right), \tilde{a}_{4}=\left(s_{1}, s_{5}\right), \tilde{a}_{5}=\left(s_{4}, s_{2}\right)$, and $W=(0.2,0.3,0.15,0.22,0.13)$. Reordering the collection $\left\{\tilde{a}_{1}, \tilde{a}_{2}, \tilde{a}_{3}, \tilde{a}_{4}, \tilde{a}_{5}\right\}$ in descending order, we get $\tilde{b}_{1}=\tilde{a}_{5}>\tilde{b}_{2}=\tilde{a}_{3}>\tilde{b}_{3}=\tilde{a}_{2}>\tilde{b}_{4}=\tilde{a}_{4}>\tilde{b}_{5}=\tilde{a}_{1}$.

- $\operatorname{iMed}\left(\tilde{a}_{1}, \tilde{a}_{2}, \tilde{a}_{3}, \tilde{a}_{4}, \tilde{a}_{5}\right)=\tilde{b}_{\frac{5+1}{2}}=\tilde{b}_{3}=\tilde{a}_{2}=\left(s_{2}, s_{4}\right)$.

- Since $u_{1}=w_{5}=0.13, u_{2}=w_{3}^{2}=0.15, u_{3}=w_{2}=0.3, u_{4}=w_{4}=0.22, u_{5}=w_{1}=0.2$, then $T_{1}=u_{1}=0.13, T_{2}=u_{1}+u_{2}=0.28, T_{3}=u_{1}+u_{2}+u_{3}=0.58>0.5$. Consequently,

$$
\operatorname{iLWM}\left(\tilde{a}_{1}, \tilde{a}_{2}, \tilde{a}_{3}, \tilde{a}_{4}, \tilde{a}_{5}\right)=\tilde{b}_{3}=\tilde{a}_{2} .
$$

Theorem 3.2. iLWM is idempotent, bounded, commutative and monotonous, i.e.:

(B1) For all $\tilde{a} \in \tilde{S}$, and $W=\left(w_{1}, \ldots, w_{n}\right)$ is a weight vector,

$$
\operatorname{iLWM}\left(\left(w_{1}, \tilde{a}\right), \ldots,\left(w_{n}, \tilde{a}\right)\right)=\tilde{a} ;
$$

(B2) For all $\tilde{a}_{i} \in \tilde{S}$, and $W=\left(w_{1}, \ldots, w_{n}\right)$ is a weight vector,

$$
\min \left(\tilde{a}_{1}, \ldots, \tilde{a}_{n}\right) \leq \operatorname{iLWM}\left(\left(w_{1}, \tilde{a}_{1}\right), \ldots,\left(w_{n}, \tilde{a}_{n}\right)\right) \leq \max \left(\tilde{a}_{1}, \ldots, \tilde{a}_{n}\right) ;
$$

(B3) For all $\tilde{a}_{i} \in \tilde{S}, W=\left(w_{1}, \ldots, w_{n}\right)$ is a weight vector, and $\sigma$ is a permutation on the set $\{1, \ldots, n\}$,

$$
\operatorname{iLWM}\left(\left(w_{1}, \tilde{a}_{1}\right), \ldots,\left(w_{n}, \tilde{a}_{n}\right)\right)=\operatorname{iLWM}\left(\left(w_{\sigma(1)}, \tilde{a}_{\sigma(1)}\right), \ldots,\left(w_{\sigma(n)}, \tilde{a}_{\sigma(n)}\right)\right) ;
$$

(B4) Let $\tilde{a}_{i}, \tilde{c}_{i} \in \tilde{S}$ such that $\tilde{a}_{i} \leq \tilde{c}_{i}$ for all $i=1, \ldots n$, and $W=\left(w_{1}, \ldots, w_{n}\right)$ is a weight vector,

$$
\operatorname{iLWM}\left(\left(w_{1}, \tilde{a}_{1}\right), \ldots,\left(w_{n}, \tilde{a}_{n}\right)\right) \leq \operatorname{iLWM}\left(\left(w_{1}, \tilde{c}_{1}\right), \ldots,\left(w_{n}, \tilde{c}_{n}\right)\right) .
$$

Proof. (B1)-(B2) are straightforward.

(B3) It is implied from the fact that the $j$-th largest of $\left\{\tilde{a}_{1}, \ldots, \tilde{a}_{n}\right\}$ is equal to the that of $\left\{\tilde{a}_{\sigma(1)}, \ldots, \tilde{a}_{\sigma(n)}\right\}$.

(B4) It is easily shown that the $j$-th largest of $\left\{\tilde{a}_{1}, \ldots, \tilde{a}_{n}\right\}$ is smaller or equal to the that of $\left\{\tilde{c}_{1}, \ldots, \tilde{c}_{n}\right\}$. So, (B4) is also proved.

\subsection{Convex combination of two intuitionistic linguistic labels}

Definition 3.2. Let $\tilde{a}_{1}$ and $\tilde{a}_{2} \in \tilde{S}$. A convex combination of $\tilde{a}_{1}$ and $\tilde{a}_{2}$ that has an associated weight vector $W=\left(w_{1}, w_{2}\right)$, denoted by $\mathrm{iC}\left\{w_{i}, \tilde{a}_{i}, i=1,2\right\}$, is defined as follows:

$$
\mathrm{iC}\left\{w_{i}, \tilde{a}_{i}, i=1,2\right\}=\tilde{s}_{k},
$$

where $k=i+\operatorname{round}\left[w_{1}(j-i)\right]$, round $(\cdot)$ is the usual round operator, and $\tilde{s}_{j}=\max \left\{\tilde{a}_{1}, \tilde{a}_{2}\right\}$, $\tilde{s}_{i}=\min \left\{\tilde{a}_{1}, \tilde{a}_{2}\right\}$.

For convenience, we also denote by $\mathrm{iC}\left\{w_{1}, w_{2}, \tilde{a}_{1}, \tilde{a}_{2}\right\}$ the expression $\mathrm{iC}\left\{w_{i}, \tilde{a}_{i}, i=1,2\right\}$.

Theorem 3.3. Let $\mathrm{iC}$ be a convex combination having an associated weight vector $W=$ $\left(w_{1}, w_{2}\right)$. We have:

(C1) For all $\tilde{a}_{1}, \tilde{a}_{2} \in \tilde{S}$, iC $\left\{1,0, \tilde{a}_{1}, \tilde{a}_{2}\right\}=\max \left(\tilde{a}_{1}, \tilde{a}_{2}\right)$ and $\mathrm{iC}\left\{0,1, \tilde{a}_{1}, \tilde{a}_{2}\right\}=\min \left(\tilde{a}_{1}, \tilde{a}_{2}\right)$;

(C2) (Idempotency) For all $\tilde{s}_{i} \in \tilde{S}$, iC $\left\{w_{1}, w_{2}, \tilde{s}_{i}, \tilde{s}_{i}\right\}=\tilde{s}_{i}$; 
(C3) (Boundary) For all $\tilde{a}_{1}, \tilde{a}_{2} \in \tilde{S}, \min \left(\tilde{a}_{1}, \tilde{a}_{2}\right) \leq \mathrm{iC}\left\{w_{i}, \tilde{a}_{i}, i=1,2\right\} \leq \max \left(\tilde{a}_{1}, \tilde{a}_{2}\right)$;

(C4) (Monotonicity) For all $\tilde{a}_{1}, \tilde{a}_{2}, \tilde{c}_{1}, \tilde{c}_{2} \in \tilde{S}$ such that $\tilde{a}_{1} \leq \tilde{c}_{1}$, and $\tilde{a}_{2} \leq \tilde{c}_{2}$,

$$
\mathrm{iC}\left\{w_{i}, \tilde{a}_{i}, i=1,2\right\} \leq \mathrm{iC}\left\{w_{i}, \tilde{c}_{i}, i=1,2\right\}
$$

(C5) (Commutativity) For all $\tilde{a}_{1}, \tilde{a}_{2} \in \tilde{S}, \mathrm{iC}\left\{w_{1}, w_{2}, \tilde{a}_{1}, \tilde{a}_{2}\right\}=\mathrm{iC}\left\{w_{1}, w_{2}, \tilde{a}_{2}, \tilde{a}_{1}\right\}$.

Proof. (C1)-(C2) and (C5) are trivial.

(C3) As round $(\cdot)$ is an increasingly monotonous function, we have

$$
i \leq i+\operatorname{round}\left[w_{1}(j-i)\right] \leq i+\operatorname{round}(j-i)=i+(j-i)=j, \forall i \leq j .
$$

Thus, $\min \left(\tilde{a}_{1}, \tilde{a}_{2}\right) \leq \mathrm{iC}\left\{w_{i}, \tilde{a}_{i}, i=1,2\right\} \leq \max \left(\tilde{a}_{1}, \tilde{a}_{2}\right)$.

(C4) Assume that $\tilde{a}_{1}=\tilde{s}_{i}, \tilde{a}_{2}=\tilde{s}_{j}, \tilde{c}_{1}=\tilde{s}_{p}$ and $\tilde{c}_{2}=\tilde{s}_{q}$ with $i \leq j$. By hypothesis, $i \leq p$ and $j \leq q$.

Case 1. $p \leq q$. We have

$$
\left(p+w_{1}(q-p)\right)-\left(i+w_{1}(j-i)\right)=\left(1-w_{1}\right)(p-i)+w_{1}(q-j) \geq 0,
$$

or

$$
i+w_{1}(j-i) \leq p+w_{1}(q-p) .
$$

By the increasing monotonicity of round $(\cdot)$ function,

$$
\mathrm{iC}\left\{w_{i}, \tilde{a}_{i}, i=1,2\right\} \leq \mathrm{iC}\left\{w_{i}, \tilde{c}_{i}, i=1,2\right\}
$$

Case 2. $p>q$. We have

$$
\mathrm{iC}\left\{w_{i}, \tilde{a}_{i}, i=1,2\right\} \leq \max \left(\tilde{a}_{1}, \tilde{a}_{2}\right)=\tilde{s}_{j} \leq \tilde{s}_{q}=\min \left(\tilde{c}_{1}, \tilde{c}_{2}\right) \leq \mathrm{iC}\left\{w_{i}, \tilde{c}_{i}, i=1,2\right\} .
$$

Using the convex combination of two intuitionistic linguistic labels, convex combination of $n$ intuitionistic linguistic labels is recursively defined as the following definition.

Definition 3.3. A convex combination of $n(n \geq 2)$ intuitionistic linguistic labels $\tilde{a}_{1}, \ldots, \tilde{a}_{n}$ in $\tilde{S}$, that has associated weight vector $W=\left(w_{1}, \ldots, w_{n}\right)$, denoted by $\mathrm{iC}^{n}\left\{w_{k}, \tilde{a}_{k}, k=1, \ldots, n\right\}$, is defined as follows:

(1) $n=2: \mathrm{iC}^{2}\left\{w_{i}, \tilde{a}_{i}, i=1,2\right\}=\mathrm{iC}\left\{w_{i}, \tilde{a}_{i}, i=1,2\right\}$,

(2) $n>2$ :

$$
\mathrm{iC}^{n}\left\{w_{k}, \tilde{a}_{k}, k=1,2, \ldots, n\right\}=\mathrm{iC}\left\{w_{1}, 1-w_{1}, \tilde{b}_{1}, \mathrm{iC}^{n-1}\left\{\frac{w_{h}}{1-w_{1}}, \tilde{b}_{h}, h=2, \ldots, n\right\}\right\}
$$

where $b_{j}$ is the $j$-th largest of $a_{i}$.

We also denote by $\mathrm{iC}^{n}\left\{w_{1}, \ldots, w_{n}, \tilde{a}_{1}, \ldots, \tilde{a}_{n}\right\}$ the expression $\mathrm{iC}^{n}\left\{w_{i}, \tilde{a}_{i}, i=1, \ldots, n\right\}$. 


\subsection{Intuitionistic linguistic ordered weighted averaging operator}

Definition 3.4. Intuitionistic linguistic ordered weighted averaging ( $\mathrm{iLOWA}_{1}$ ) operator, whose weight vector is $W=\left(w_{1}, \ldots, w_{n}\right)$, is defined as follows:

$$
\operatorname{iLOWA}_{1 W}\left(\tilde{a}_{1}, \ldots, \tilde{a}_{n}\right)=\mathrm{iC}^{n}\left\{w_{k}, \tilde{a}_{k}, k=1, \ldots, n\right\} .
$$

Example 3.3. Let $\tilde{a}_{1}=\left(s_{0}, s_{4}\right), \tilde{a}_{2}=\left(s_{2}, s_{4}\right), \tilde{a}_{3}=\left(s_{3}, s_{1}\right), \tilde{a}_{4}=\left(s_{1}, s_{5}\right), \tilde{a}_{5}=\left(s_{4}, s_{2}\right)$ be intuitionistic linguistic labels belonging to the intuitionistic label set given in Example 2.3, and $W=(0.2,0.3,0.15,0.22,0.13)$. It is easy to see that (Table 1) $\tilde{a}_{1}=\tilde{s}_{2}, \tilde{a}_{2}=\tilde{s}_{8}, \tilde{a}_{3}=\tilde{s}_{20}$, $\tilde{a}_{4}=\tilde{s}_{3}, \tilde{a}_{5}=\tilde{s}_{21}$. So, $\tilde{b}_{1}=\tilde{s}_{21}, \tilde{b}_{2}=\tilde{s}_{20}, \tilde{b}_{3}=\tilde{s}_{8}, \tilde{b}_{4}=\tilde{s}_{3}, \tilde{b}_{5}=\tilde{s}_{2}$. We have:

$$
\begin{aligned}
\mathrm{iLOWA}_{1 W}\left(\tilde{a}_{1}, \tilde{a}_{2}, \tilde{a}_{3}, \tilde{a}_{4}, \tilde{a}_{5}\right) & =\mathrm{iC}\left\{\frac{20}{100}, \frac{80}{100}, \tilde{s}_{21}, \mathrm{iC}^{4}\left\{\frac{30}{80}, \frac{15}{80}, \frac{22}{80}, \frac{13}{80}, \tilde{s}_{20}, \tilde{s}_{8}, \tilde{s}_{3}, \tilde{s}_{2}\right\}\right\} ; \\
\mathrm{iC}^{4}\left\{\frac{30}{80}, \frac{15}{80}, \frac{22}{80}, \frac{13}{80}, \tilde{s}_{20}, \tilde{s}_{8}, \tilde{s}_{3}, \tilde{s}_{2}\right\} & =\mathrm{iC}\left\{\frac{30}{80}, \frac{50}{80}, \tilde{s}_{20}, \mathrm{iC}^{3}\left\{\frac{15}{50}, \frac{22}{50}, \frac{13}{50}, \tilde{s}_{8}, \tilde{s}_{3}, \tilde{s}_{2}\right\}\right\} ; \\
\mathrm{iC}^{3}\left\{\frac{15}{50}, \frac{22}{50}, \frac{13}{50}, \tilde{s}_{8}, \tilde{s}_{3}, \tilde{s}_{2}\right\} & =\mathrm{iC}\left\{\frac{15}{50}, \frac{35}{50}, \tilde{s}_{8}, \mathrm{iC}^{2}\left\{\frac{22}{35}, \frac{13}{35}, \tilde{s}_{3}, \tilde{s}_{2}\right\}\right\} .
\end{aligned}
$$

Let us calculate $\mathrm{iC}^{2}\left\{\frac{22}{35}, \frac{13}{35}, \tilde{s}_{3}, \tilde{s}_{2}\right\}$, substitute in to (4):

$$
\begin{aligned}
\mathrm{iC}^{2}\left\{\frac{22}{35}, \frac{13}{35}, \tilde{s}_{3}, \tilde{s}_{2}\right\} & =\tilde{s}_{2+\operatorname{round}\left[\frac{22}{35}(3-2)\right]=\tilde{s}_{3} ;} \\
\mathrm{iC}^{3}\left\{\frac{15}{50}, \frac{22}{50}, \frac{13}{50}, \tilde{s}_{8}, \tilde{s}_{3}, \tilde{s}_{2}\right\} & =\mathrm{iC}\left\{\frac{15}{50}, \frac{35}{50}, \tilde{s}_{8}, \tilde{s}_{3}\right\}=\tilde{s}_{3+\operatorname{round}\left[\frac{15}{50}(8-3)\right]=\tilde{s}_{5} ;} \\
\mathrm{iC}^{4}\left\{\frac{30}{80}, \frac{15}{80}, \frac{22}{80}, \frac{13}{80}, \tilde{s}_{20}, \tilde{s}_{8}, \tilde{s}_{3}, \tilde{s}_{2}\right\} & =\mathrm{iC}\left\{\frac{30}{80}, \frac{50}{80}, \tilde{s}_{20}, \tilde{s}_{5}\right\}=\tilde{s}_{5+\operatorname{round}\left[\frac{30}{80}(20-5)\right]=\tilde{s}_{11} ;} \\
\mathrm{iLOWA}_{1 W}\left(\tilde{a}_{1}, \tilde{a}_{2}, \tilde{a}_{3}, \tilde{a}_{4}, \tilde{a}_{5}\right) & =\mathrm{iC}\left\{\frac{20}{100}, \frac{80}{100}, \tilde{s}_{21}, \tilde{s}_{11}\right\}=\tilde{s}_{11+\operatorname{round}\left[\frac{20}{100}(21-11)\right]}=\tilde{s}_{13} .
\end{aligned}
$$

Theorem 3.4. iLOWA 1 operator satisfies the following properties:

(D1) (Idempotency) For all $\tilde{a} \in \tilde{S}$, and $W=\left(w_{1}, \ldots, w_{n}\right)$ is a weight vector,

$$
\operatorname{iLOWA}_{1 W}(\tilde{a}, \ldots, \tilde{a})=\tilde{a} ;
$$

(D2) (Boundary) For all $\tilde{a}_{i} \in \tilde{S}$, and $W=\left(w_{1}, \ldots, w_{n}\right)$ is a weight vector,

$$
\min _{i=1, \ldots, n}\left\{\tilde{a}_{i}\right\} \leq \operatorname{iLOWA}_{1 W}\left(\tilde{a}_{1}, \ldots, \tilde{a}_{n}\right) \leq \max _{i=1, \ldots, n}\left\{\tilde{a}_{i}\right\}
$$

(D3) (Monotonicity) Let $\tilde{a}_{i}, \tilde{c}_{i} \in \tilde{S}$ such that $\tilde{a}_{i} \leq \tilde{c}_{i}$ for all $i=1, \ldots, n$, and $W=$ $\left(w_{1}, \ldots, w_{n}\right)$ is a weight vector,

$$
\operatorname{iLOWA}_{1 W}\left(\tilde{a}_{1}, \ldots, \tilde{a}_{n}\right) \leq \operatorname{iLOWA}_{1 W}\left(\tilde{c}_{1}, \ldots, \tilde{c}_{n}\right) ;
$$

(D4) (Commutativity) For all $\tilde{a}_{i} \in \tilde{S}, \pi$ is a permutation over the set of arguments, and $W=\left(w_{1}, \ldots, w_{n}\right)$ is a weight vector

$$
\operatorname{iLOWA}_{1 W}\left(\tilde{a}_{1}, \ldots, \tilde{a}_{n}\right)=\operatorname{iLOWA}_{1 W}\left(\pi\left(\tilde{a}_{1}\right), \ldots, \pi\left(\tilde{a}_{n}\right)\right) .
$$


Proof. Properties (D1), (D2) and (D4) are clearly verified because of the corresponding properties of the convex combination of two intuitionistic linguistic labels. We prove (D3) by induction on $n$.

- It is true for $n=2$, because in this case $\mathrm{iLOWA}_{1}$ is reduced to iC.

- For all $t \geq 2$, suppose that it is true for $n=t$. i.e.:

$$
\operatorname{iLOWA}_{1 W}\left(\tilde{a}_{1}^{\prime}, \ldots, \tilde{a}_{t}^{\prime}\right) \leq \operatorname{iLOWA}_{1 W}\left(\tilde{c}_{1}^{\prime}, \ldots, \tilde{c}_{t}^{\prime}\right),
$$

for all $\tilde{a}_{i}^{\prime}, \tilde{c}_{i}^{\prime} \in \tilde{S}$ such that $\tilde{a}_{i}^{\prime} \leq \tilde{c}_{i}^{\prime}$ for all $i=1, \ldots, t$, and $W^{\prime}=\left(w_{1}^{\prime}, \ldots, w_{t}^{\prime}\right)$ is a weight vector.

Let us consider $\tilde{a}_{i}, \tilde{c}_{i} \in \tilde{S}$ such that $\tilde{a}_{i} \leq \tilde{c}_{i}$ for all $i=1, \ldots, t+1$, and $W=\left(w_{1}, \ldots, w_{t+1}\right)$ is a weight vector. Without loss of generality we may assume: that $\tilde{a}_{1} \geq \ldots \geq \tilde{a}_{t+1}$ and $\tilde{c}_{1} \geq \ldots \geq \tilde{c}_{t+1}$. Then,

$$
\operatorname{iLOWA}_{1 W}\left(\tilde{a}_{1}, \ldots, \tilde{a}_{t+1}\right)=\mathrm{iC}\left\{w_{1}, 1-w_{1}, \tilde{a}_{1}, \mathrm{iC}^{t}\left\{\frac{w_{h}}{1-w_{1}}, \tilde{a}_{h}, h=2, \ldots, t+1\right\}\right\},
$$

and

$$
\operatorname{iLOWA}_{1 W}\left(\tilde{c}_{1}, \ldots, \tilde{c}_{t+1}\right)=\mathrm{iC}\left\{w_{1}, 1-w_{1}, \tilde{c}_{1}, \mathrm{iC}^{t}\left\{\frac{w_{h}}{1-w_{1}}, \tilde{c}_{h}, h=2, \ldots, t+1\right\}\right\} .
$$

By (5),

$$
\mathrm{iC}^{t}\left\{\frac{w_{h}}{1-w_{1}}, \tilde{a}_{h}, h=2, \ldots, t+1\right\} \leq \mathrm{iC}^{t}\left\{\frac{w_{h}}{1-w_{1}}, \tilde{c}_{h}, h=2, \ldots, t+1\right\} .
$$

Using the monotonicity of the $\mathrm{iC}$ operator, inequality $(6)$ and $\tilde{a}_{1} \leq \tilde{c}_{1}$, we obtain:

$$
\begin{aligned}
& \mathrm{iC}\left\{w_{1}, 1-w_{1}, \tilde{a}_{1}, \mathrm{iC}^{t}\left\{\frac{w_{h}}{1-w_{1}}, \tilde{a}_{h}, h=2, \ldots, t+1\right\}\right\} \\
\leq & \mathrm{iC}\left\{w_{1}, 1-w_{1}, \tilde{c}_{1}, \mathrm{iC}^{t}\left\{\frac{w_{h}}{1-w_{1}}, \tilde{c}_{h}, h=2, \ldots, t+1\right\}\right\} .
\end{aligned}
$$

Thus, $\operatorname{iLOWA}_{1 W}\left(\tilde{a}_{1}, \ldots, \tilde{a}_{t+1}\right) \leq \operatorname{iLOWA}_{1 W}\left(\tilde{c}_{1}, \ldots, \tilde{c}_{t+1}\right)$. (D3) is proved.

\section{AGGREGATION OPERATORS FOR CONTINUOUS INTUITIONISTIC LABEL SET}

In this section, we extend the discrete intuitionistic labels set $\tilde{S}=\left\{\left(s_{i}, s_{j}\right) \in S^{2} \mid i+j \leq g\right\}$ to continuous one, such that the indexes of the membership and the nonmembership of each intuitionistic linguistic label take continuous values.

Definition 4.1. Continuous intuitionistic linguistic label set, $\hat{S}$, is defined as the set of all pair $\hat{a}=\left(s_{\alpha}, s_{\beta}\right)$ such that $\alpha, \beta \geq 0$ and $0 \leq \alpha+\beta \leq g$, i.e.:

$$
\hat{S}=\left\{\left(s_{\alpha}, s_{\beta}\right) \mid \alpha, \beta \geq 0, \alpha+\beta \leq g\right\} .
$$

Each $\hat{a}=\left(s_{\alpha}, s_{\beta}\right)$ is termed as a continuous intuitionistic linguistic label. Two continuous intuitionistic linguistic labels $\left(s_{\alpha}, s_{\beta}\right),\left(s_{\delta}, s_{\sigma}\right)$ are equal, denoted by $\left(s_{\alpha}, s_{\beta}\right)=\left(s_{\delta}, s_{\sigma}\right)$, if $\alpha=\delta$ and $\beta=\sigma$. 
In Definition 4.1, it is not required that $\left(s_{\alpha}, s_{\beta}\right) \in S^{2}$, i.e., $\alpha$ and $\beta$ can take non-integer values. For each $\hat{a} \in \hat{S}$, if $\hat{a} \in \tilde{S}, \hat{a}$ is called a original intuitionistic label; otherwise, a virtual intuitionistic label. The score, confidence degree, relations " $\geq$ ", and " $>$ " of continuous intuitionistic label are defined similarly to the those of (discrete) intuitionistic label (Definitions 2.2, 2.3).

Definition 4.2. Let $\left\{\hat{a}_{1}, \ldots, \hat{a}_{n}\right\}$ be a collection of continuous intuitionistic labels, $\hat{a}_{j}=\left(s_{\alpha_{j}}, s_{\beta_{j}}\right)$ for all $j=1, \ldots, n$. Intuitionistic linguistic averaging (iLA $\mathrm{A}_{2}$ ) operators is defined as:

$$
\operatorname{iLA}_{2}\left(\hat{a}_{1}, \ldots, \hat{a}_{n}\right)=\left(s_{\bar{\alpha}}, s_{\bar{\beta}}\right),
$$

where $\bar{\alpha}=\left(\sum_{j=1}^{n} \alpha_{j}\right) / n$, and $\bar{\beta}=\left(\sum_{j=1}^{n} \beta_{j}\right) / n$.

Theorem 4.1. For all $\left\{\hat{a}_{1}, \ldots, \hat{a}_{n}\right\}$ be a collection of continuous intuitionistic labels in $\hat{S}$, $\mathrm{iLA}_{2}\left(\hat{a}_{1}, \ldots, \hat{a}_{n}\right)$ is an continuous intuitionistic linguistic label.

Proof. Since $\hat{a}_{j}=\left(s_{\alpha_{j}}, s_{\beta_{j}}\right)$ is an intuitionistic linguistic label, we have $\alpha_{j} \geq 0, \beta_{j} \geq 0$, and $\alpha_{j}+\beta_{j} \leq g$, for all $j=1, \ldots, n$. It implies $\bar{\alpha} \geq 0, \bar{\beta} \geq 0$, and

$$
\bar{\alpha}+\bar{\beta}=\frac{\sum_{j=1}^{n}\left(\alpha_{j}+\beta_{j}\right)}{n} \leq(n g) / g=g,
$$

which proves the theorem.

Definition 4.3. Let $\left\{\hat{a}_{1}, \ldots, \hat{a}_{n}\right\}$ be a collection of continuous intuitionistic labels in $\hat{S}, \hat{a}_{j}=$ $\left(s_{\alpha_{j}}, s_{\beta_{j}}\right)$ for all $j=1, \ldots, n$, and $W=\left(w_{1}, \ldots, w_{n}\right)$ be a weight vector of $\left(\hat{a}_{1}, \ldots, \hat{a}_{n}\right)$. Intuitionistic linguistic weighted averaging $\left(\mathrm{iLWA}_{2}\right)$ operator is defined as:

$$
\operatorname{iLWA}_{2}\left(\left\langle w_{1}, \hat{a}_{1}\right\rangle, \ldots,\left\langle w_{n}, \hat{a}_{n}\right\rangle\right)=\left(s_{\bar{\alpha}}, s_{\bar{\beta}}\right)
$$

where $\bar{\alpha}=\sum_{j=1}^{n} w_{j} \alpha_{j}$, and $\bar{\beta}=\sum_{j=1}^{n} w_{j} \beta_{j}$.

Theorem 4.2. For all $\left\{\hat{a}_{1}, \ldots, \hat{a}_{n}\right\}$ be a collection of continuous intuitionistic labels in $\hat{S}$ and $W=\left(w_{1}, \ldots, w_{n}\right)$ be a weight vector of $\left(\hat{a}_{1}, \ldots, \hat{a}_{n}\right), \mathrm{iLWA}_{2}\left(\left\langle w_{1}, \hat{a}_{1}\right\rangle, \ldots,\left\langle w_{n}, \hat{a}_{n}\right\rangle\right)$ is an continuous intuitionistic linguistic label.

Proof. Similar to Theorem 4.1.

Definition 4.4. Let $\left\{\hat{a}_{1}, \ldots, \hat{a}_{n}\right\}$ be set of continuous intuitionistic labels in $\hat{S}$, and $\hat{b}_{j}=\left(s_{\delta_{j}}, s_{\sigma_{j}}\right)$ be the $j$-th largest of $\hat{a}_{i}$. Intuitionistic linguistic ordered weighted aggregation (iLOWA $2 \mathrm{~W}$ ) operator, whose associated weight vector is $W=\left(w_{1}, \ldots, w_{n}\right)$, is defined as:

$$
\operatorname{iLOWA}_{2 \mathrm{~W}}\left(\hat{a}_{1}, \ldots, \hat{a}_{n}\right)=\left(s_{\bar{\delta}}, s_{\bar{\sigma}}\right) \text {, }
$$

where $\bar{\delta}=\sum_{j=1}^{n} w_{j} \delta_{j}$, and $\bar{\sigma}=\sum_{j=1}^{n} w_{j} \sigma_{j}$.

Theorem 4.3. For all $\left\{\hat{a}_{1}, \ldots, \hat{a}_{n}\right\}$ be a collection of continuous intuitionistic labels in $\hat{S}$ and $W=\left(w_{1}, \ldots, w_{n}\right)$ be a weight vector, $\operatorname{iLOWA}_{2 \mathrm{~W}}\left(\hat{a}_{1}, \ldots, \hat{a}_{n}\right)$ is a continuous intuitionistic linguistic label.

Proof. Similar to Theorem 4.1.

Example 4.1. Consider intuitionistic linguistic labels $\hat{a}_{1}=\left(s_{1.2}, s_{4.6}\right), \hat{a}_{2}=\left(s_{2.3}, s_{3.1}\right), \hat{a}_{3}=$ $\left(s_{2.4}, s_{1.9}\right), \hat{a}_{4}=\left(s_{1.3}, s_{4.1}\right), \hat{a}_{5}=\left(s_{2.2}, s_{0.7}\right)$ in $\hat{S}=\left\{\left(s_{\alpha}, s_{\beta}\right) \mid \alpha, \beta \geq 0, \alpha+\beta \leq 6\right\}$, and a 
weight vector $W=(0.19,0.15,0.23,0.31,0.12)$. We have $\hat{b}_{1}=\hat{a}_{5}>\hat{b}_{2}=\hat{a}_{3}>\hat{b}_{3}=\hat{a}_{2}>\hat{b}_{4}=$ $\hat{a}_{4}>\hat{b}_{5}=\hat{a}_{1}$. Then,

$$
\begin{aligned}
& \operatorname{iLA}_{2}\left(\hat{a}_{1}, \ldots, \hat{a}_{5}\right)=\left(s_{\frac{1.2+2.3+2.4+1.3+2.2}{5}}, s_{\frac{4.6+3.1+1.9+4.1+0.7}{5}}\right)=\left(s_{2.08}, s_{2.88}\right), \\
& \operatorname{iLWA}_{2}\left(\left\langle w_{1}, \hat{a}_{1}\right\rangle, \ldots,\left\langle w_{5}, \hat{a}_{5}\right\rangle\right)=\left(s_{0.19 \times 1.2+0.15 \times 2.3+0.23 \times 2.4+0.31 \times 1.3+0.12 \times 2.2}\right. \text {, } \\
& s_{0.19 \times 4.6+0.15 \times 3.1+0.23 \times 1.9+0.31 \times 4.1+0.12 \times 0.7)} \\
& =\left(s_{1.792}, s_{3.131}\right) \text {, } \\
& \mathrm{iLOWA}_{2 \mathrm{~W}}\left(\hat{a}_{1}, \ldots, \hat{a}_{5}\right)=\left(s_{0.19 \times 2.2+0.15 \times 2.4+0.23 \times 2.3+0.31 \times 1.3+0.12 \times 1.2},\right. \\
& \left.s_{0.19 \times 0.7+0.15 \times 1.9+0.23 \times 3.1+0.31 \times 4.1+0.12 \times 4.6)}\right) \\
& =\left(s_{1.854}, s_{2.954}\right) \text {. }
\end{aligned}
$$

Remark 4.1. It is easily seen that $\mathrm{iLA}_{2}, \mathrm{iLWA}_{2}$, and iLOWA 2 are idempotent, bounded, increasingly monotonous and commutative.

\section{AN APPLICATION}

Zhang etal. ( [31], algorithm (I)) proposed the intuitionistic fuzzy linguistic preference relation (IFLPR) as a new type of preference relation. Then, a GDM problem under intuitionistic linguistic information was considered, in which, the 2-tuple representation was utilized to represent intuitionistic linguistic information. In this paper, a refined approach to this problem is introduced, which generates the same result as [31]. This approach avoids the use of 2-tuple representation. Thus, it seem be simpler and more understandable.

Using IFLPRs, intuitionistic linguistic averaging ( $\mathrm{iLA}_{2}$ ) and intuitionistic linguistic weighted averaging $\left(\mathrm{iLWA}_{2}\right)$ operators, the method is described as follows:

Step 1. Consider a GDM problem, where $X=\left\{x_{1}, \ldots, x_{n}\right\}$ is a set of alternatives, and $D=\left\{d_{1}, \ldots, d_{m}\right\}$ is a set of DMs. Assume that $W=\left(w_{1}, \ldots, w_{m}\right)$ is the weight vector of DMs, $w_{h}$ is the weight of $D_{h}(h=1, \ldots, m)$. Denote $P^{h}=\left[p_{i j}^{h}\right]_{n \times n}$, where $p_{i j}^{h}$ is the intuitionistic linguistic preference of alternative $x_{i}$ over the alternative $x_{j}$, given by the $\mathrm{DM} d_{h}(i, j=1, \ldots, n$, $h=1, \ldots, m)$.

Step 2. Utilize the iLWA 2 operator to aggregate the $P^{h}=\left[p_{i j}^{h}\right]_{n \times n}(h=1, \ldots, n)$, and derive a collective IFLPR $P=\left[p_{i j}\right]_{n \times n}$, where

$$
p_{i j}=\mathrm{iLWA}_{2}\left(\left\langle w_{1}, p_{i j}^{1}\right\rangle, \ldots,\left\langle w_{m}, p_{i j}^{m}\right\rangle\right),(i, j=1, \ldots, n) .
$$

Step 3. For each alternative $x_{i}$, we get an overall collective intuitionistic linguistic label:

$$
p_{i}=\operatorname{iLA}_{2}\left(p_{i 1}, \ldots, p_{i n}\right),(i=1, \ldots, n) .
$$

Step 4. Rank all $p_{i}$ by means of the order relation defined in Definition 2.3, then rank all the alternatives $x_{i}$ and select the best one(s) in accordance with the values of $p_{i}(i=1, \ldots, n)$.

Example 5.1. Consider the linguistic label set $S$ which consists of seven linguistic labels: $s_{0}=$ certain, $s_{1}=$ extremely_likely, $s_{2}=$ meaningful_chance, $s_{3}=i t_{-}$may, $s_{4}=$ small_chance, $s_{5}=$ extremely_unlikely, $s_{6}=$ impossible and its corresponding intuitionistic label set $\tilde{S}$. There 
are two DMs who use intuitionistic labels to access the preferences over the set of alternatives $X=$ $\left\{x_{1}, x_{2}, x_{3}, x_{4}\right\}$.

- DM $p_{1}$ has his/her weight $w_{1}=0.6$ [31]:

$$
P^{1}=\left[\begin{array}{llll}
\left(s_{3}, s_{3}\right) & \left(s_{1}, s_{2}\right) & \left(s_{2}, s_{2}\right) & \left(s_{1}, s_{3}\right) \\
\left(s_{2}, s_{1}\right) & \left(s_{3}, s_{3}\right) & \left(s_{2}, s_{3}\right) & \left(s_{1}, s_{4}\right) \\
\left(s_{2}, s_{2}\right) & \left(s_{3}, s_{2}\right) & \left(s_{3}, s_{3}\right) & \left(s_{3}, s_{3}\right) \\
\left(s_{3}, s_{1}\right) & \left(s_{4}, s_{1}\right) & \left(s_{3}, s_{3}\right) & \left(s_{3}, s_{3}\right)
\end{array}\right] .
$$

- $\mathrm{DM} p_{2}$ has his/her weight $w_{2}=0.4[31]$ :

$$
P^{2}=\left[\begin{array}{llll}
\left(s_{3}, s_{3}\right) & \left(s_{2}, s_{4}\right) & \left(s_{4}, s_{1}\right) & \left(s_{5}, s_{0}\right) \\
\left(s_{4}, s_{2}\right) & \left(s_{3}, s_{3}\right) & \left(s_{4}, s_{2}\right) & \left(s_{6}, s_{0}\right) \\
\left(s_{1}, s_{4}\right) & \left(s_{2}, s_{4}\right) & \left(s_{3}, s_{3}\right) & \left(s_{3}, s_{3}\right) \\
\left(s_{0}, s_{5}\right) & \left(s_{0}, s_{6}\right) & \left(s_{3}, s_{3}\right) & \left(s_{3}, s_{3}\right)
\end{array}\right]
$$

Use (7) to aggregate $P^{1}$ and $P^{2}$, we obtain the collective IFLPR:

$$
P=\left[\begin{array}{cccc}
\left(s_{3}, s_{3}\right) & \left(s_{1.4}, s_{2.8}\right) & \left(s_{2.8}, s_{1.6}\right) & \left(s_{2.6}, s_{1.8}\right) \\
\left(s_{2.8}, s_{1.4}\right) & \left(s_{3}, s_{3}\right) & \left(s_{2.8}, s_{2.6}\right) & \left(s_{3}, s_{2.4}\right) \\
\left(s_{1.6}, s_{2.8}\right) & \left(s_{2.6}, s_{2.8}\right) & \left(s_{3}, s_{3}\right) & \left(s_{3}, s_{3}\right) \\
\left(s_{1.8}, s_{2.6}\right) & \left(s_{2.4}, s_{3}\right) & \left(s_{3}, s_{3}\right) & \left(s_{3}, s_{3}\right)
\end{array}\right]
$$

Use (8), we get $p_{1}=\left(s_{2.45}, s_{2.3}\right), p_{2}=\left(s_{2.9}, s_{2.35}\right), p_{3}=\left(s_{2.55}, s_{2.9}\right), p_{4}=\left(s_{2.55}, s_{2.9}\right)$. Then, $x_{2}>x_{1}>x_{3}=x_{4}$.

\section{CONCLUSIONS}

In this paper, many operators, which can be used to aggregate intuitionistic linguistic information, are defined. A modified algorithm based on linguistic labels representation is given to solve the GDM problem with IFLPR. The results of the modification are the same as the previous method based on 2 -tuple intuitionistic linguistic representation [31], and this proves the correctness of the refinement.

\section{ACKNOWLEDGMENT}

The authors are greatly indebted to anonymous reviewers for their comments and their valuable suggestions that improved the quality and clarity of the paper. This work is sponsored by the NAFOSTED under contract No. 102.01-2015.01.

\section{REFERENCES}

[1] K. T. Atanassov, "Intuitionistic fuzzy sets", Fuzzy Sets and Systems, vol. 20, pp. 87-96, 1986.

[2] K. T. Atanassov and S. Stoeva, "Intuitionistic L-fuzzy sets", in Cybernetics and Systems Research (Eds. R. Trappl), Elsevier Science Pub., Amsterdam, vol. 2, pp. 539-540, 1986.

[3] G. Bordogna, M. Fedrizzi, and G. Passi, "A linguistic modeling of consensus in group decision making based on OWA operator", IEEE Transactions on Systems, Man, and Cybernetics, vol. 27, pp. 126-132, 1997. 
[4] F. Chiclana, F. Herrera, and E. Herrera-Viedma, "Integrating multiplicative preference relations in a multipurpose decision-making model based on fuzzy preference relations", Fuzzy Sets and Systems, vol. 122, pp. 277-291, 2001.

[5] B. C. Cuong and P. H. Phong, "Max - Min Composition of Linguistic Intuitionistictic Fuzzy Relations and Application in Medical Diagnosis", VNU Journal of Science: Comp. Science $\mathcal{E}$ Com. Eng., vol. 30, no. 4, pp. 601-968, 2014.

[6] Y. Q. Dai, Z. S. Xu, and Q. L. Da, "New evaluation scale of linguistic information and its application", Chinese Journal of Management Science, vol. 16, no. 2, pp. 145-349, 2008.

[7] M. Delgado, J. L. Verdegay, and M. A. Vila, "On aggregation operations of linguistic labels", International Journal of Intelligent Systems, vol. 8, pp. 351-370, 1993.

[8] M. Delgado, F. Herrera F, and E. Herrera-Viedma, "Combining numerical and linguistic information in group decision making", Information ciences, vol. 107, pp. 177-194, 1998.

[9] F. Herrera and J. L. Verdegay "Linguistic assessments in group decision", in Proceedings of the First European Congress on Fuzzy and Intelligent Technologies, Aachen, pp. 941-948, 1993.

[10] F. Herrera and E. Herrera-Viedma, "Aggregation operators for linguistic weighted information", IEEE Transactions on Systems, Man, and Cybernetics-Part A, vol. 27, no. 5, pp. 646-656, 1997.

[11] F. Herrera and L. Martínez, "A 2-tuple fuzzy linguistic representation model for computing with words", IEEE Transactions on Fuzzy Systems, vol. 8, pp. 746-752, 2000.

[12] F. Herrera and L. Martínez, "An approach for combining linguistic and numerical information based on the 2-tuple fuzzy linguistic representation model in decision-making", International Journal of Uncertainty, Fuzziness and Knowledge-Based Systems, vol. 8, pp. 539-562, 2000.

[13] F. Herrera and E. Herrera-Viedma, "Choice functions and mechanisms for linguistic preference relations", European Journal of Operational Research, vol. 120, pp. 144-161, 2000.

[14] F. Herrera and E. Herrera-Viedma, "Linguistic decision analysis: steps for solving decision problems under linguistic information", Fuzzy Sets and Systems, vol. 115, pp. 67-82, 2000.

[15] F. Herrera, S. Alonso, F. Chiclana, and E. Herrera-Viedma, "Computing with words in decision making: foundations, trends and prospects", Fuzzy Optimization and Decision Making, vol. 8, no. 4, pp. 337-364, 2009.

[16] L. Martinez, D. Ruan, and F. Herrera, "Computing with Words in Decision support Systems: An overview on Models and Applications", International Journal of Computational Intelligence Systems, vol. 3, no. 4, pp. 382-395, 2010.

[17] P. H. Phong and B. C. Cuong, "Some intuitionist linguistic aggregation operators", Journal of Computer Science and Cybernetics, vol. 30, no. 3, pp. 216-226, 2014.

[18] V. Torra, "The weighted OWA operator", International Journal of Intelligent Systems, vol. 12, pp. 153-166, 1997. 
[19] T. Tanino, "Fuzzy Preference Relations in Group Decision Making", in Non-conventional Preference Relations in Decision Making, J. Kacprzyk and M. Roubens (Eds.), Springer-Verlag, Berlin, pp. 54-71, 1988.

[20] T. Tanino, "On Group Decision Making Under Fuzzy Preferences". in Multiperson Decision Making Using Fuzzy Sets and Possibility Theory, J. Kacprzyk and M. Fedrizzi (Eds.), Kluwer Academic Publishers, Dordrecht, pp. 172-185, 1990.

[21] M. M. Xia and Z. .S. Xu, "Managing hesitant information in GDM problems under fuzzy and multiplicative preference relations", International Journal of Uncertainty, Fuzziness and Knowledge-based Systems, vol. 21, pp. 865-897, 2013.

[22] Z. S. Xu, "Uncertain Multiple Attribute Decision Making: Methods and Applications", Tsinghua University Press, Beijing, 2004.

[23] Z. S. Xu, "EOWA and EOWG operators for aggregating linguistic labels based on linguistic preference relations", International Journal of Uncertainty, Fuzziness and Knowledge-Based Systems, vol. 12. pp. 791-810, 2004.

[24] Z. S. Xu, "A method based on linguistic aggregation operators for group decision making with linguistic preference relations", Information Sciences, vol. 166, pp. 19-30, 2004.

[25] Z. S. Xu, "On generalized induced linguistic aggregation operators", International Journal of General Systems, vol. 35, pp. 17-28, 2006.

[26] Z. S. Xu, "Intuitionistic preference relations and their application in group decision making", Information Sciences, vol. 177, pp. 2363-2379, 2007.

[27] Z. S. Xu, "Group decision making based on multiple types of linguistic preference relations", Information Sciences, vol. 178, pp. 452-467, 2008.

[28] R. R. Yager, "A new methodology for ordinal multiobjective decisions based on fuzzy sets", Decision Sciences, vol. 12, pp. 589-600, 1981.

[29] R. R. Yager, "Applications and extensions of OWA aggregations", International Journal of Man-Machine Studied, vol. 37, pp. 103-132, 1992.

[30] L. A. Zadeh, "The concept of a linguistic variable and its application to approximate reasoning - I", Information Scences, vol. 8, pp. 199-249, 1975.

[31] Y. Zhang, H. X. Ma, B. H. Liu, and J. Liu, "Group decision making with 2-tuple intuitionistic fuzzy linguistic preference relations", Soft Computing, vol. 16, pp. 1439-1446, 2012.

Received on March 23 - 2015

Revised on June 09 - 2016 